\title{
Decision Issues on Part Supply in Build-to-Order Manufacturing Systems
}

\begin{abstract}
Ki-Seok Choi ${ }^{\dagger}$
Dept. of Industrial and Management Engineering, Hankuk University of Foreign Studies

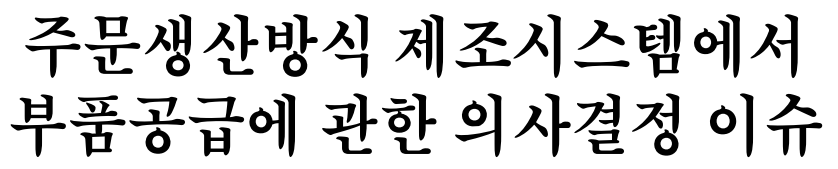

최 기 석

한국외국어대학교 산업경영공학과

In Build-to-Order manufacturing systems, products are usually manufactured by a pull system. The manufacturers order only what they need when it is needed. The suppliers are expected to deliver parts as much as needed. When the part demand fluctuates significantly, this places lots of challenges on the suppliers. In this letter, we explore the decision issues regarding part supplies for Build-to-Order manufacturing systems, especially in the auto industry, and suggest how to address them in order to make the manufacturing systems work more efficiently.
\end{abstract}

Keywords: Build-to-Order, Supply Chain, Pull System

\section{Introduction}

Inspired by the success of Dell Computers, Build-to-Order (BTO) has become a powerful and growing trend in the electronics industry. One competitive advantage from BTO is speed. Quickly converting customer orders into delivered products helps win and satisfy customers. It also reduces workin-process inventories with their demands on capital and space, which in turn reduces the risks of obsolescence, and helps speed new products to market. Finally, reduced inventories and shortened order to delivery cycles reduce the cash-tocash cycle improving return on invested capital and helping to sustain profits even when margins shrink. A second competitive advantage from BTO is product variety-being able to offer each customer his own personalized product. The goal of BTO is to achieve personalization at mass-production prices by eliminating finished goods inventory. But the challenge is formidable. Personalization means unpredictable variability in demand spread over a product mix as diverse as the market itself. The challenge of BTO is to manage that variability without the traditional cushion of finished goods inventory. In this letter, we study what challenges the part suppliers have faced in BTO manufacturing systems. We also identify issues and problems in the supply chain, which are closely related to part supply in BTO manufacturing systems.

\section{Challenges to Part Suppliers}

The industry has pursued several strategies in its quest for

이 연구는 한국외국어대학교 교내학술연구비의 지원에 의해 이루어진 것임.

† Corresponding author : Professor Ki-Seok Choi, Dept. of Industrial and Management Engineering, Hankuk University of Foreign Studies, Youngin 449-791, Korea, Tel :+82-31-330-4253, Fax :+82-31-330-4093, E-mail : kchoi@hufs.ac.kr

Received December 30, 2012; Accepted January 8, 2013. 
BTO. Flexible manufacturing techniques, for example, reduce setup time, enhance cross-training and employ more versatile tooling. Information technology offers hope in the form of collaborative forecasting and sharing of production schedules. These efforts have helped, but the industry still struggles with variability and the bullwhip effect (Cachon, 1999; Chen et al., 2000; Lee et al., 1997) still lashes suppliers.

Since it is not possible to schedule far in advance, BTO requires that production be organized according to a pull system in which the flow of material is triggered first by customer orders and thereafter by downstream consumption. Lean manufacturing, pioneered by Toyota Motor Company in Japan during the 1950s, has become the standard pull production system in the automotive industry. Along with other philosophical principles of manufacturing practice, lean manufacturing promotes just-in-time principles that call for ordering only what is needed when it is needed. To support this practice, each supplier must deliver the anticipated usage of each part on a regular schedule, in some cases as often as several times per day.

Significant variation in scheduled deliveries poses another challenge for transportation, as it is difficult to plan efficient loads and routes when the volumes to be moved change dramatically from day to day. When volumes are unusually large, the excess is often sent via premium freight. When volumes are down, vehicles travel half-empty. Since a major manufacturer has roughly tons of freight in transit at any given time, small inefficiencies in transportation have a significant financial impact.

Several factors conspire to increase day-to-day variations in part usage. First, manufacturers are increasingly producing more than one model on a single assembly line. Although daily production at an assembly plant may be relatively constant, the mix of products changes from one day to the next. Second, as manufacturers are moving toward BTO, they are driving down order-to-delivery cycles and reducing the banks of sequenced orders released to plants from weeks to days.

Combined, these factors produce variations in day-to-day part usage and add several percentage points to the cost of parts and a lot of freight cost. Further, these large swings in demand force suppliers to carry significant finished goods inventory, finance excess production capacity or risk shutting down the manufacturer's lines during periods of peak demand.

\section{Order Quantity}

Following the principles of lean manufacturing, manufacturers tend to make daily or even more frequent orders for ex- actly the parts that are needed for scheduled production until the next order. The trends in the industry discussed in the previous section have caused the order quantities, or the number of parts requested with each order release, to vary dramatically from day to day. The more consistently a manufacturer makes its orders, the more it can reduce freight costs. Further, with more consistent orders, suppliers should realize savings in their operations, which they should pass on in the form of lower part prices.

In fact, by damping the variations in order quantities to its first tier suppliers, a manufacturer should benefit from reductions in the bullwhip effect all the way up its supply chain. It is conceivable that if each tier adopts this operating philosophy and protects its suppliers from residual variability in its own demands, the bullwhip can be eliminated.

It is important to find the right balance between inventory and order variability. The manufacturer has part inventory fed by a stream of shipments from the supplier. When the inventory grows too large, the manufacturer may temporarily interrupt or reduce the order quantity. When it falls too low, the manufacturer may increase or expedite shipments. Manufacturers manage their parts supplies by holding a small inventory and meet varying demands by changing the order quantities frequently. To ensure that the part does not run out, the manufacturer specifies a minimum inventory level for the part and strives to maintain at least that amount on site. Since customer demand is random, the inventory level at the manufacturer's site will on rare occasions drop below the minimum level. These rare events may be brought on by temporary disruptions in the supplier's production or in transportation. The manufacturer and supplier may agree on a fixed delivery or order frequency. The manufacturer's challenge in this issue is to minimize the costs associated with holding the inventory and the operational costs involved in adjusting the shipment sizes or order quantities.

\section{Minimum Inventory Level}

In a BTO environment, the manufacturer needs to fulfill customer orders quickly. An important performance measure for the manufacturer is the so called fill rate, the percentage of orders fulfilled on time. Achieving a high fill rate requires ample production capacity and high availability of parts. The manufacturer should set a high minimum inventory level to prevent part shortages, and thus to improve on-time delivery of customer orders. On the other hand, setting the minimum level too high incurs the costs and space requirements of excess inventory and robs the supplier of flexibility. To reduce 
supply chain costs, it makes sense to keep the minimum inventory level as small as possible while protecting the manufacturer's service level.

Occasionally the inventory level of a part will drop below the minimum level. These exceptions, hopefully rare, do occur. There are many factors, some controllable and some not, that cause them. For example, they may be due to a sudden surge of customer orders, transportation delays, shipment errors or production problems at the supplier. A paper by Choi et al. (2004) revealed that, in a capacitated production environment, the fill rate depends on which of these exceptions occurs. For example, maintaining an inventory above the minimum level most of the time does not guarantee a high fill rate if the supplier's production is not reliable. Suppose, for example, that a supplier cannot ship for $k$ consecutive periods. This might arise because of extreme weather, quality problems, etc. The manufacturer's maximum production in a period is limited by its capacity, $c$-the number of units the manufacturer can produce in a period when all the necessary parts are available. This implies that $c$ is the minimum inventory necessary for the production process to operate without interruption due to part shortages in a single period. Thus, keeping the inventory level above $c k$ allows the manufacturer to operate normally even during an interval of $k$ consecutive periods without shipments. Setting the minimum inventory this way, however, simply costs too much. Manufacturer needs more cost effective methods for setting minimum levels that adequately guarantee his target fill rate.

\section{Delivery Route}

In the auto industry, parts are generally large and relatively inexpensive, say in comparison to the electronics industry. In-bound freight represents a significant cost and the opportunities for consolidation and coordination are significant. As a consequence auto manufacturers take a more active role in in-bound transportation and recruit Lead Logistics Providers (LLP's) to help coordinate shipments.

An LLP helps a manufacturer coordinate shipments from the three-to-four thousand suppliers who serve it, by identifying and exploiting opportunities for consolidation and coordination of flows. Typically, an LLP relies on tools forged in the build-to-stock era to provide that support. Those tools focus on managing the average flows, not on managing the variability. To buffer against the fluctuations, analysts either inflate the volumes or reduce the capacities of the transportation assets or both. These can, however, at best poorly reflect the complex influences of variability. In the end, us- ing them guarantees that poor average capacity utilization and excess expedited shipments will simultaneously plague the network.

An analysis is required which incorporate explicitly the variability inherent in BTO. For example, in building regularly scheduled routes that pick up parts from several suppliers, traditional models ensure that the sum of the average volumes from the suppliers on the route does not exceed the capacity of the vehicle. By considering the variances and covariances in these volumes it may be possible to build routes with higher capacity utilization, lower variance in the overall volumes and better control of the probability that the volumes on the routes exceed the capacity of the vehicle. Natural approaches to explore include explicitly modeling the variance in the volume on each lane of the planned in-bound network and using it to incorporate appropriate penalties representing the expected costs of expediting. Such models would naturally be large, possibly non-linear, mixed integer programs and one obvious challenge would be to devise effective heuristics.

\section{Other Issues}

A typical automotive assembly plant receives about 3,000 different parts and has limited space and budget to allocate among them. Thus, the manufacturer needs to find the optimal allocation of scarce resources among the competing parts. One of the principles of lean manufacturing is the notion of footprinting or clearly identifying the floor space allocated to each part or function. One consequence of this philosophy is that floor space is dedicated to a single use. Manufacturers must not only allocate limited floor space, but also a limited budget among competing parts.

While there are numerous extensions and generalizations (Baita et al., 1998; Bertazzi and Speranza, 1999; Blumenfeld et al., 1987, Ernst and Pyke, 1993; Hahm and Yano, 1992; Jackson et al., 1988; Speranza and Ukovich, 1994) all models for determining the optimal order frequency are essentially based on some elaboration of the simple EOQ relationship. But order frequency can also impact the stochastic component of inventory at the manufacturer's site. One key factor influencing the choice of order frequency is the distance between the supplier and the manufacturer. For high complexity parts that come in a variety of colors or with a choice of features, distance also influences the accuracy of the manufacturer's forecasted usage. The manufacturer freezes the production sequence for an amount of fixed horizon. Beyond that, it can only forecast usage and must rely on alternative 
mechanisms for getting the parts in the right sequence. Hence distance plays a more significant role in deciding frequency and that role involves both the deterministic and the stochastic elements. In deciding order frequency it is necessary to consider the distance.

\section{Summary}

In this letter, we review the operation-level decision issues for managing part supply inherent in the BTO manufacturing systems. These issues are closely related to the variance in demand for a part, the resources allocated to managing the part and the total cost of supplying it. In order to accomplish the efficiency which the BTO intends originally, the supply chain including the part supplier should be well designed and managed with careful attentions to the operational decisions such as part order quantity, minimum inventory level, and delivery route.

\section{References}

Baita, F., Ukovich, W., Pesenti, R., and Favaretto, D. (1998), Dynamic
Routing-and-Inventory Problems : A Review, Transportation Research-A, 32, 585-598.

Bertazzi, L. and Speranza, M. G. (1999), Minimizing Logistics Costs in Multistage Supply Chains, Naval Research Logistics, 46, 399-417.

Blumenfeld, D. E., Burns, L. D., Daganzo, C. F., Frick, M. C., and Hall, R. W. (1987), Reducing Logistics Costs at General Motors, Interfaces, 17, 26-47.

Cachon, G. P. (1999), Managing Supply Chain Demand Variability with Scheduled Ordering Policies, Management Science, 45, 843-856.

Chen, F., Drezner, Z., Ryan, J. K., and Simchi-Levi, D. (2000), Quantifying the Bullwhip Effect in a Simple Supply Chain : The Impact of Forecasting, Lead Times, and Information, Management Science, 46, 436-444

Choi, K.-S., Dai, J. G., and Song, J. S. (2004), On Measuring Supplier Performance under Vendor-Managed-Inventory Programs, Manufacturing and Service Operations Management, 6, 53-72.

Ernst, R. and Pyke, D. F. (1993), Optimal Base Stock Policies and Truck Capacity in a Two-Echelon System, Naval Research Logistics, 40, 879-903.

Hahm, J. and Yano, C. A. (1992), The Economic Lot and Delivery Scheduling Problem : The Single Item Case, International Journal of Production Economics, 28, 235-252.

Jackson, P. L., Maxwell, W. L., and Muckstadt, J. A. (1988), Determining Optimal Reorder Intervals in Capacitated Production-Distribution Systems, Management Science, 34, 938-958.

Lee, H. L., Padmanabhan, V., and Whang, S. (1997), The Paralyzing Curse of the Bullwhip Effect in a Supply Chain, Sloan Management Review, 38, 93-102.

Speranza, M. G. and Ukovich, W. (1994), Minimizing Transportation and Inventory Costs for Several Products on a Single Link, Operations Research, 42, 879-894. 